\title{
Cultura organizacional inspirada en valores como cuarto eje de la sostenibilidad: un modelo de responsabilidad social*
}

\section{Organizational culture inspired on values as fourth pillar of sustainability: a social responsibility model}

Recibido: 22 de enero de 2014 Revisado: 22 de marzo de 2014 Aceptado: 27 de junio de 2014

Stella León Ordoñe****

Congregación Paulinas

Amanda Quintana Caro****

Microhard SAS

\section{RESUMEN}

El presente artículo describe los principales aspectos que se tuvieron en cuenta para la construcción de un Modelo de Responsabilidad Social (MRS) dirigido a la Congregación Paulinas, organización religiosa que muestra su compromiso con este tema de gran importancia por sus repercusiones e implicaciones a nivel mundial.
La investigación parte de entender el comportamiento actual de Paulinas frente a la Responsabilidad Social (RS), para lo cual se identifican los grupos de interés más importantes de la Congregación, a quienes se les aplica un instrumento que permite recopilar información primaria. El diagnóstico situacional permite la construcción del MRS para Paulinas, basado en tres enfoques fundamentales: el Enfoque Apreciativo (EA), el Enfoque Axiológico y

\footnotetext{
* Artículo de investigación.

** Correspondencia: Stella León Ordoñez. Licenciada en Teología con especialización en Espiritualidad. Directora de Talento Humano Congregación Paulinas. Correo electrónico: stellaleon@gmail.com

****Amanda Quintana Caro. Ingeniera Electrónica. Especialista en redes de alta velocidad y distribuidas. Gerente de Operaciones Microhard SAS. Correo electrónico: amandaquintana@yahoo.com
} 
el de Resultados de Aprendizajes Esperados (RAE). Desde lo metodológico se integra el método Paulino "Verdad, Camino y Vida" como aspecto fundamental de la institución. Desde lo normativo se enmarca en los Principios de RS contenidos en la guía técnica ISO 26000:2010, en el ciclo PHVA y en un eje planteado como cultura organizacional inspirada en valores, que se propone como un cuarto eje específico y transversal para el modelo actual de sostenibilidad basado en el concepto de Desarrollo Sostenible. La implementación del modelo se apoya en la elaboración de una cartilla pedagógica, sencilla y de fácil comprensión, con la cual se realiza una prueba piloto; el modelo resultante puede ser aplicado en todas las organizaciones que compartan o se identifiquen con la misión y visión Paulinas.

Palabras clave: enfoque apreciativo, cultura organizacional, responsabilidad social empresarial, resultados de aprendizaje esperados.

\section{ABSTRACT}

This article describes the main aspects that were considered for the construction of a Social Responsibility Model (MRS) aimed at Pauline Congregation, religious organization that shows your commitment to this issue, of great importance for their implications and impact on a global level.

This research starts understanding the current behavior on Pauline related to of the social responsibility (SR). Therefore, an instrument is applied to the major interest groups of the Congregation that have been identified, in order to collect primary information. The diagnosis enables the construction of the SR model for the Pauline Congregation, based on three main approaches: the Appreciative Inquiry Approach, the Axiological Approach and the
Expected Learning Outcomes. From the methodological, the Pauline method "Truth Way and Life", as a fundamental aspect of the institution, is integrated. From the normative view, is framed on the Principles of Social Responsibility found in ISO 26000:2010, on the PDCA cycle and also raised on an axis described as organizational culture inspired on values, which is proposed as a fourth specific and transverse axis for the sustainability based on the concept of Sustainable Development. The implementation of the model is based on developing a guide that is pedagogical, simple and easy to understand, with which a pilot test is made. This model could be applied to all organizations which share or identify with the Pauline's mission and vision.

Keywords: Appreciative inquiry approach, corporate social responsibility, expected learning outcomes, organizational culture.

\section{INTRODUCCIÓN}

La Congregación Paulinas Bogotá, dedicada al anuncio del evangelio, ofrece diversos servicios sociales tales como: educación no formal, producción editorial, difusión del evangelio, misiones y pastoral, entre otros. No obstante que la Congregación orienta su gestión hacia el cumplimiento cabal de todas sus responsabilidades sociales, no ha estructurado un sistema de RS que le permita evaluar si su comportamiento respecto de la $\mathrm{RS}$ es acertado; por ello se hace indispensable comprobar de forma práctica el cabal cumplimiento de los principios establecidos en la Guía de Responsabilidad Social ISO 26000:2010, en los servicios que presta. De la misma manera, no obstante su orientación hacia la búsqueda permanente de un comportamiento socialmente responsable, la no implementación de un sistema de RS hace que no se pueda verificar dentro 
del marco de RS y como congregación católica, el compromiso, la proyección e incidencia de Paulinas en la sociedad o en los grupos de la comunidad en la que presta sus servicios evangelizadores.

Con el desarrollo de la investigación se pretende posicionar a Paulinas como Institución religiosa líder en el tema de RS, logrando varios propósitos tales como: generar conciencia al interior de la comunidad sobre la urgente necesidad de asumir la RS como proyecto integral en el desarrollo de la institución, lograr conciliación entre la vida laboral y la familia, aplicar medidas que promuevan un mejor clima organizacional a nivel interno con repercusiones externas que influyan en el medio ambiente y el entorno, proyectar a Paulinas en una visión que vaya más allá de acciones sociales puntuales y de poca incidencia, y establecer el desarrollo de prácticas sociales para favorecer el crecimiento de sus diferentes grupos de interés.

Se plantea como objetivo general construir un Modelo de Responsabilidad Social empresarial (MRS) para la Congregación Paulinas-Bogotá, cuyo proceso de estructuración detecte posibles inconvenientes para su implementación y diseñe un modelo pedagógico que los mitigue o controle.

Igualmente, se plantea la siguiente hipótesis fenomenológica con el fin de contrastarla en el transcurso del trabajo de investigación:

Paulinas-Bogotá evidencia un comportamiento empresarialmente responsable aislado, no en su conjunto, que hace necesaria la construcción de un MRS a implementar en el futuro.

Sin desconocer que son muchos los autores que hacen mención a las teorías de RS, la investigación se desarrolla en el marco de dos enfoques: modernidad y modernización de Consuelo Corredor y mínimos éticos de Adela Cortina. Corredor expresa que:
La modernización se refiere al proceso de apropiación de la naturaleza por el hombre, con el fin de desarrollar las fuerzas productivas y poder disponer de una mayor riqueza. La modernidad se refiere a la apropiación del hombre de su propia naturaleza, lo que significa hacer de los individuos no solo sujetos transformadores de su entorno material, sino también, y principalmente, sujetos de su propia transformación. (Corredor y Misas, 2001, p. 19)

Cortina (1998) plantea respecto de la Responsabilidad Social Empresarial, una ética cívica en donde la empresa se entiende como modelo comunitario y a su vez, describe las claves de la nueva cultura empresarial, basada en los mínimos éticos que toda organización debe cumplir. Con base en esto, las organizaciones deben focalizar sus modelos de empresa y promover valores que fomenten una cultura empresarial con sentido comunitario, que favorezcan la convivencia social, la solidaridad y el desarrollo; es decir, que permita, lo que Consuelo Corredor denomina como modernidad: "transformación de la persona y de su entorno", para que sea completo el proceso de desarrollo. Estos dos enfoques, dan una clara orientación a la investigación para colocar como base unos principios éticos mínimos o axiológicos que promuevan no solo la convivencia, sino que aporten a la transformación de las personas y de su entorno.

Para dar transcendencia a esta visión de la ética es de suma importancia abordar los planteamientos que la enseñanza social (doctrina) de la Iglesia Católica hace frente al desarrollo y sus impactos. Algunos documentos que se refieren al tema son: la encíclica Rerum Novarum, del Papa León XIII, escrita en 1892, en la que plantea la calidad humana; la encíclica Quadragesimo Anno, Pio XI; Mater et Magistra y Pacem in Terris, Juan XXIII; Populorum Progressio y Gaudium et Spes, Pablo VI; Centesimus Anmus, Juan Pablo II, entre otros. Sin embargo, se hace énfasis en la encíclica Caritas in Veritate, en la que el Papa Benedicto XVI (2009) plantea una doctrina específica sobre la RS de las empresas para aplicarla a la cooperación y al 
desarrollo, que implica como el Papa expresa: "en estos tiempos de dificultades del desarrollo debidas a la globalización y agravadas por la crisis económica financiera no se pueden olvidar los principios tradicionales de la ética social, como la transparencia, la honestidad y la responsabilidad, que constituyen una exigencia de la razón económica misma"(p. 36).

Por último, dentro del marco teórico, se consideran los tratados y acuerdos internacionales en relación con la RS tales como el Pacto Global, la Declaración de Río sobre el medio ambiente y el desarrollo y la Declaración de la Organización Internacional del Trabajo - OIT, relativa a los principios y derechos fundamentales en el trabajo. En el marco normativo se tiene en cuenta la guía internacional ISO 26000:2010.

\section{METODOLOGÍA}

Metodológicamente esta investigación se fundamenta en el constructivismo social. Berger y Luckmann plantean: "La realidad se construye socialmente. $\mathrm{La}$ realidad es una cualidad propia de los fenómenos que reconocemos como independientes de nuestra propia volición (no podemos "hacerlos desaparecer") y el conocimiento es la certidumbre de que los fenómenos son reales y que poseen características específicas" (2001, p. 13). Hammersley y Atkinson por su parte destacan: "Toda investigación social se basa en la capacidad humana de realizar observación participante. Actuamos en el mundo social y somos capaces de vernos a nosotros y nuestras acciones como objetos de ese mundo. Al incluir nuestro propio papel dentro del foco de investigación y explotar sistemáticamente nuestra participación como investigadores en el mundo que estamos estudiando, podemos desarrollar y comprobar la teoría sin tener que hacer llamamientos inútiles al empirismo ya sea en su variedad naturalista o positivista” (1994, p. 40).
En lo referente a la creación del conocimiento, este se construye por el propio acto de investigación, "Lo real está constituido por el acto de conocer, en vez de una percepción objetiva del mundo" (Le Moigne, 1995, pp. 71-72). Para construir dicho conocimiento se emplea el método del enfoque apreciativo (EA), denominado método de las 4D's, por sus palabras en inglés, Discover, Dream, Design, Destiny, que dentro de la investigación se denominan descubrimiento, deseo/ sueño, diseño y destino.

El EA se entiende entonces como la búsqueda cooperativa y co-evolucionaria de lo mejor de la gente, sus organizaciones y el mundo a su alrededor, "Lo cual envuelve descubrimientos sistemáticos de aquello que da vida a una organización o una comunidad cuando es más efectiva y capaz en términos económicos, ecológicos y humanos" (Cooperrider y Whitney, 2005, p. 7), es decir, cuando sus procesos o actividades se adelantan con RS. En este sentido, la intervención en una organización o comunidad a través del EA:

[...] da lugar a la indagación, imaginación e innovación. En lugar de negociación, crítica y diagnóstico en espiral, hay un descubrimiento, deseo, diseño y destino. El EA envuelve el arte y práctica de formular preguntas incondicionalmente positivas que fortalecen las capacidades del sistema para aprender, anticipar y elevar el potencial positivo. Por medio de la indagación movilizada de las masas, cientos y aún miles de personas pueden verse envueltas en la co-creación colectiva del futuro. El EA asume que cada organización y comunidad tiene muchos caminos para lo positivo: lo que la gente habla sobre sus capacidades pasadas, presentes y futuras, o el corazón positivo. El EA conecta el conocimiento y energía de tal corazón directamente con la organización o la agenda de cambio de una organización, y cambios jamás pensados como posibles de repente se movilizan democráticamente (Cooperrider y Whitney, 2005, p. 9).

El EA se orienta por varios principios teóricos, identificados por Varona (2007), que inspiran, justifican 
y guían la práctica de la intervención apreciativa: el principio de simultaneidad, el principio poético, el principio imaginario/anticipatorio, el principio afirmativo/positivo, el principio del poder de la pregunta, el principio de la sinergia, el principio del ejemplo y el principio de libertad para elegir.

Según el alcance 0 grado de profundidad es una investigación comprensiva que integra aportes del constructivismo, en particular del constructivismo social, al resaltar la diaria construcción del devenir de la organización Paulinas-Bogotá, constituida por las redes, por los ciclos e historias que en ella se viven a diario, las que no solo hacen que su realidad sea creada continuamente sino que, además, existan múltiples realidades dentro de ella. Según el método utilizado, es una investigación cualitativa que fundamentada en los principios teóricos de la fenomenología parte de un fenómeno, se procede a la recolección y toma de datos, y posteriormente, a través de la metodología del EA, se analizan dichos datos y se generan unas conclusiones.

Se utiliza en el método cualitativo un "diálogo", que no es otra cosa que una entrevista semiestructurada, que se aplica a una muestra significativa de hermanas y laicos que representan diferentes niveles de autoridad dentro de la institución, y que posteriormente, y dado que lo que se requiere es contrastar la hipótesis fenomenológica, utiliza la Encuesta Apreciativa, previamente elaborada, que se aplica a una muestra representativa de los miembros de los grupos de interés, como instrumento del método de las 4D’s que hacen parte del EA.

Sobre la Encuesta Apreciativa Varona señala: "[...] este tipo de encuesta supone el arte de hacer preguntas apreciativas que estimulen el potencial positivo que existe en las personas y en las organizaciones. La encuesta apreciativa es el corazón de la intervención apreciativa" (2007, pp. 407-408).
Por tanto, para la encuesta, se prepara un cuestionario de preguntas teniendo en cuenta cada una de las fases del EA, esto es, descubrimiento, deseo, diseño, destino, y la técnica que se emplea, es un formato escrito que las incluye, que no pasa de una página y que se entrega a las personas para que cada una de ellas lo responda -sin la intervención de un encuestador- y lo envíe a través de un correo institucional en el término de dos días a partir del momento que se recibe.

Una vez se recopilan los formatos que incluyen las preguntas de la encuesta, la sistematización de las respuestas se realiza así: todas las respuestas a cada una de las preguntas de los grupos de interés encuestados, se tabulan en una plantilla en Excel por pregunta y por grupo de interés y con base en los objetivos propuestos, enmarcadas en los ejes de la RS dentro del enfoque del Desarrollo Sostenible: eje social, eje ambiental y eje económico o productivo, dentro de la Congregación, se elabora un filtro para establecer las constantes como valor y también aquellas prácticas contrarias al valor, con lo cual se procede, en primer lugar, a identificar y a reunir el conjunto de valores, cualidades y atributos encontrados en las encuestas, que definen tanto a un grupo de interés como a la Institución misma. Estos datos obtenidos se reúnen en una herramienta denominada "rejilla", elaborada por las autoras de la investigación, en la que se relacionan las preguntas de la encuesta con las cuatro fases del enfoque apreciativo o 4D’s, el ciclo PHVA y los ejes social, ambiental y económico de la sostenibilidad, como finalidad de la RS. Asimismo, se determina para cada uno de estos ejes, tanto a nivel personal como institucional, lo que se está y lo que no se está haciendo bien y las razones de ello, y se procede, en segundo lugar, a promulgar el cambio que se debe lograr y el compromiso que se adquiere frente a cada uno de estos aspectos a través de proposiciones provocativas que se construyen y elaboran, bajo la rigurosidad y criterios propuestos definidos por Hammond (1996). Paralelamente, se plantean los 
objetivos de la investigación como resultados de aprendizaje esperados (RAE) de acuerdo a la taxonomía de Bloom cuyo propósito es establecer lo que se espera que los diferentes grupos de interés sepan, comprendan, y sean capaces de demostrar frente a la RS.

Sobre los RAE, Kennedy indica que se puede considerar como una buena definición operativa la presentada en el Manual del Sistema Europeo de Transferencia de Créditos: "Los RAE son enunciados con respecto a lo que se espera que el estudiante sea capaz de hacer, comprender y/o sea capaz de demostrar una vez terminado un proceso de aprendizaje. El proceso de aprendizaje puede ser, por ejemplo, una clase, un módulo o un programa completo" (Kennedy, 2007, p. 19). La Tabla 1 presenta los objetivos de la investigación planteados como Resultados de Aprendizaje Esperados (RAE) según la taxonomía de Bloom en el dominio cognitivo y en los niveles 1. Conocimiento y 6. Evaluación.

Tabla 1. Ejemplo de los objetivos de la investigación como RAE

\begin{tabular}{|c|c|c|}
\hline TEMA & RAE & ACTIVIDAD DE EVALUACIÓN \\
\hline $\begin{array}{l}\text { Diagnóstico del comportamiento } \\
\text { social, ambiental y económico de } \\
\text { Paulinas }\end{array}$ & $\begin{array}{l}\text { Describir la realidad de la Congregación en } \\
\text { cuanto a su comportamiento social, ambiental } \\
\text { y económico. }\end{array}$ & $\begin{array}{l}\text { Enumere las acciones socialmente responsables de } \\
\text { Paulinas referentes a lo ambiental, social y económico. }\end{array}$ \\
\hline Valores y principios de Paulinas & $\begin{array}{l}\text { Rememorar los principios y valores que } \\
\text { orientan a Paulinas. }\end{array}$ & $\begin{array}{l}\text { Relacione los principios y valores de Paulinas con la } \\
\text { (s) actividad (es) que usted realiza. }\end{array}$ \\
\hline $\begin{array}{l}\text { Grupos de Interés con los que } \\
\text { interactúa Paulinas }\end{array}$ & $\begin{array}{l}\text { Reconocer los grupos de interés de interacción } \\
\text { con Paulinas y su compromiso de RS. }\end{array}$ & $\begin{array}{l}\text { Defina los grupos de interés de RS de interacción con } \\
\text { Paulinas. }\end{array}$ \\
\hline Principios de la Norma ISO 26000 & $\begin{array}{l}\text { Identificar los elementos de RS de la norma } \\
\text { ISO } 26000 .\end{array}$ & $\begin{array}{l}\text { Relacione los principios de la norma ISO } 26000 \text { afines } \\
\text { con Paulinas. }\end{array}$ \\
\hline $\begin{array}{l}\text { Modelo de responsabilidad social } \\
\text { Paulinas-Bogotá }\end{array}$ & Presentar el modelo de RS de Paulinas-Bogotá. & $\begin{array}{l}\text { Enumere las innovaciones que el modelo de RS le } \\
\text { aporta a Paulinas-Bogotá. }\end{array}$ \\
\hline $\begin{array}{l}\text { Cartilla pedagógica basada en el } \\
\text { modelo de RS para Paulinas-Bogotá }\end{array}$ & $\begin{array}{l}\text { Dar a conocer el modelo de RS de Paulinas- } \\
\text { Bogotá a través de una cartilla. }\end{array}$ & $\begin{array}{l}\text { Narre los principales impactos de RS presentados en } \\
\text { la cartilla pedagógica. }\end{array}$ \\
\hline $\begin{array}{l}\text { Diagnóstico del comportamiento } \\
\text { social, ambiental y económico de } \\
\text { Paulinas }\end{array}$ & $\begin{array}{l}\text { Evaluar críticamente el comportamiento social, } \\
\text { ambiental y económico de Paulinas. }\end{array}$ & $\begin{array}{l}\text { Valore el comportamiento social, ambiental y } \\
\text { económico de Paulinas y haga explícitos los criterios } \\
\text { con los cuales usted emite esta valoración. }\end{array}$ \\
\hline Valores y principios de Paulinas & $\begin{array}{l}\text { Argumentar por qué estos valores y principios } \\
\text { identifican la misión de Paulinas. }\end{array}$ & $\begin{array}{l}\text { Califique la proyección de estos valores y principios de } \\
\text { Paulinas en las diferentes actividades que realiza. }\end{array}$ \\
\hline $\begin{array}{l}\text { Grupos de Interés con los que } \\
\text { interactúa Paulinas }\end{array}$ & $\begin{array}{l}\text { Revisar atentamente el comportamiento y la } \\
\text { proyección social, ambiental y económica de } \\
\text { los grupos de interés con los que interactúa } \\
\text { Paulinas. }\end{array}$ & $\begin{array}{l}\text { Interprete el comportamiento de RS de los grupos } \\
\text { de interés en relación con el modelo propuesto por } \\
\text { Paulinas. }\end{array}$ \\
\hline Principios de la Norma ISO 26000 & $\begin{array}{l}\text { Evaluar la integración de los principios de la } \\
\text { norma con los propuestos en el modelo de } \\
\text { Paulinas. }\end{array}$ & $\begin{array}{l}\text { Haga un paralelo con los principios planteados en la } \\
\text { norma y los propuestos por Paulinas. }\end{array}$ \\
\hline $\begin{array}{l}\text { Modelo de responsabilidad social } \\
\text { Paulinas-Bogotá }\end{array}$ & $\begin{array}{l}\text { Medir el alcance del modelo de RS de Paulinas } \\
\text { Bogotá. }\end{array}$ & $\begin{array}{l}\text { Prediga mediante una tabla de medición el alcance } \\
\text { que puede tener el modelo. }\end{array}$ \\
\hline $\begin{array}{l}\text { Cartilla pedagógica basada en el } \\
\text { modelo de RS para Paulinas-Bogotá }\end{array}$ & $\begin{array}{l}\text { Determinar las ideas fuerza que deja la } \\
\text { propuesta pedagógica como compromiso de RS. }\end{array}$ & $\begin{array}{l}\text { Seleccione las } 2 \text { ideas fuerza de la propuesta que } \\
\text { considere las más importantes y realice un slogan. }\end{array}$ \\
\hline
\end{tabular}

Fuente: elaboración propia. 
Se realiza un análisis de la información obtenida -el consolidado de las encuestas- desde el EA y se contrasta la hipótesis fenomenológica planteada. Con base en este análisis, el marco teórico y en los objetivos como RAE, se procede a construir el Modelo de Responsabilidad Social (MRS) para la congregación Paulinas-Bogotá. Por último, se diseña una herramienta pedagógica que facilite su futura implementación 0 haga posible poner a marchar el MRS construido.

Hay necesidad de aclarar que estos fenómenos son particulares, así como la elaboración del diseño del MRS para la Congregación Paulinas-Bogotá, y que por tanto solo es válido para esta institución. No obstante, el método o el enfoque seguido, posteriormente puede ser tenido en cuenta en futuras investigaciones.

Paulinas cuenta con un equipo humano conformado por 55 religiosas de las cuales 30 se encuentran en Bogotá, con un rango de edad entre los 30 y los 80 años, también cuenta con 120 colaboradores distribuidos en el país, de los cuales en Bogotá se encuentran 75, con un rango de edad entre los 20 a los 50 años y un grupo de 130 cooperadores voluntarios de los cuales 60 prestan su servicio en la capital. El promedio de formación de todos se encuentra entre la media profesional; el $70 \%$ del personal son mujeres. Las obras apostólicas que se llevan a cabo son la Editorial Multimedial, el Centro de Comunicación Social, el Centro de Espiritualidad y los Centros de Difusión conocidos como Librerías Paulinas.

\section{RESULTADOS Y DISCUSIÓN}

El desarrollo del primer momento de la investigación en el que se realizan Entrevistas en Profundidad -diálogos- a laicos y hermanas que representan diferentes niveles de autoridad, permite la identificación a nivel general de paradigmas, tomados como creencias o mitos, a favor o de resistencia frente a la RS en Paulinas. Algunos de ellos se muestran en la Tabla 2.

Tabla 2. Algunos paradigmas, tomados como creencias o mitos, frente a la RS en Paulinas

\begin{tabular}{|c|c|}
\hline A FAVOR & DE RESISTENCIA \\
\hline $\begin{array}{l}\text { La RS es interesante puesto que } \\
\text { muestra una imagen diferente de } \\
\text { la Institución }\end{array}$ & $\begin{array}{l}\text { La gente no se interesa por } \\
\text { ese tema }\end{array}$ \\
\hline $\begin{array}{l}\text { A Paulinas le interesa no } \\
\text { solamente obtener recursos para } \\
\text { la misión que realiza, sino que } \\
\text { además contribuye a un cambio } \\
\text { de la sociedad, la reafirma como } \\
\text { Institución sin ánimo de lucro }\end{array}$ & $\begin{array}{l}\text { ¿Qué aporta concretamente } \\
\text { la RS? }\end{array}$ \\
\hline $\begin{array}{l}\text { En este sentido se puede } \\
\text { proceder a tener productos para } \\
\text { llegar a otros nichos de mercado }\end{array}$ & $\begin{array}{l}\text { Eso ya lo hacemos en la } \\
\text { evangelización }\end{array}$ \\
\hline $\begin{array}{l}\text { Posibilita e Involucra a la } \\
\text { institución en un compromiso } \\
\text { personal, institucional y mundial, } \\
\text { que abre nuevos horizontes }\end{array}$ & Es pérdida de tiempo \\
\hline $\begin{array}{l}\text { La RS expresa una imagen más } \\
\text { solidaria, al servicio de la vida }\end{array}$ & $\begin{array}{l}\text { Eso es de interés para } \\
\text { algunos, para otros no }\end{array}$ \\
\hline \multirow{2}{*}{$\begin{array}{l}\text { Ayuda a un compromiso de } \\
\text { mayor impacto y también es } \\
\text { inversión }\end{array}$} & $\begin{array}{l}\text { Es un proceso más que se } \\
\text { va a iniciar }\end{array}$ \\
\hline & $\begin{array}{l}\text { Hay algunas acciones de RS } \\
\text { pero desarticuladas y, por lo } \\
\text { tanto, no tienen engranaje } \\
\text { con lo que se hace }\end{array}$ \\
\hline
\end{tabular}

Fuente: elaboración propia.

Estos resultados son los que permiten formular posteriormente, las preguntas correspondientes a la Encuesta Apreciativa.

El segundo momento, es la identificación de los grupos de interés de Paulinas-Bogotá y la elaboración del diagnóstico, se tienen en cuenta o se acogen las descripciones formuladas en el Manual de Relación STH Accountability (Krick, Forstater, Monaghan y Sillanpää, 2006), y a partir de estas descripciones y de los diálogos o entrevistas semiestructuradas con las directivas de las 
diferentes áreas apostólicas de la Institución, las personas con posibilidades de influenciar el desempeño de la institución y los grupos afectados por las operaciones de la misma, se genera un listado de categorías de grupos de interés según la interacción que con ellos se tiene; en la Tabla 3 se observan estas categorías.

Tabla 3. Grupos de interés

\begin{tabular}{|c|c|}
\hline $\begin{array}{l}\text { CATEGORÍA } \\
\text { DE GRUPO DE } \\
\text { INTERÉS }\end{array}$ & SUBGRUPOS Y DEFINICIÓN \\
\hline Hermanas & $\begin{array}{l}\text { Gobierno Provincial (Alta Dirección): } \\
\text { compuesto por la Superiora provincial, } \\
\text { consejeras y ecónoma; Directora general } \\
\text { de apostolado; Directoras de área/ } \\
\text { departamento (6) y Hermanas de apoyo. }\end{array}$ \\
\hline \multirow[b]{2}{*}{ Colaboradores } & $\begin{array}{l}\text { Retribuidos: Son los laicos que apoyan } \\
\text { la misión de Paulinas y son retribuidos } \\
\text { según la ley. }\end{array}$ \\
\hline & $\begin{array}{l}\text { En alianza: Son las personas, grupos o } \\
\text { instituciones que colaboran con Paulinas } \\
\text { y su misión, pueden o no recibir una } \\
\text { ofrenda por ello. }\end{array}$ \\
\hline Cooperadores & $\begin{array}{l}\text { Son las personas que hacen parte de una } \\
\text { asociación de voluntarios que viven la } \\
\text { misión y espiritualidad Paulina. }\end{array}$ \\
\hline \multirow[t]{2}{*}{ Destinatarios } & $\begin{array}{l}\text { Clientes internos: Hermanas y } \\
\text { colaboradores y cooperadores. }\end{array}$ \\
\hline & $\begin{array}{l}\text { Clientes externos: Iglesia, colegios y } \\
\text { comunidades religiosas, instituciones } \\
\text { religiosas, familias, fieles en general. }\end{array}$ \\
\hline \multirow{4}{*}{ Proveedores } & Proveedores de materia prima. \\
\hline & $\begin{array}{l}\text { Proveedores de producto terminado: } \\
\text { Otras editoriales que proveen libros, } \\
\text { proveedores de artículos religiosos, } \\
\text { proveedores de DVD's, etc. }\end{array}$ \\
\hline & Autores de libros. \\
\hline & Cantautores católicos. \\
\hline
\end{tabular}

Fuente: elaboración propia.

Se adelanta en el tercer momento, el diseño, prueba piloto y aplicación de la encuesta apreciativa orientada a RS para obtener información de primera mano. La encuesta diseñada se observa en la Tabla 4.

Tabla 4. Encuesta

\begin{tabular}{|c|l|}
\hline 1. & $\begin{array}{l}\text { Describa una época de Paulinas en la que usted considere } \\
\text { estuvo totalmente comprometido con la organización, se } \\
\text { sintió "vivo" y vibrante dentro de ella. }\end{array}$ \\
\hline 2. & $\begin{array}{l}\text { ¿Cuáles aspectos son lo mejor de usted mismo como } \\
\text { colaborador en Paulinas? }\end{array}$ \\
\hline 3. & $\begin{array}{l}\text { ¿Cuáles aspectos son lo mejor de su trabajo en la } \\
\text { Institución? }\end{array}$ \\
\hline 4. & ¿Cuáles aspectos son lo mejor de la Congregación Paulinas? \\
\hline 5. & $\begin{array}{l}\text { ¿Considera usted que la Institución funcionó mejor en } \\
\text { el pasado o que por el contrario funciona mejor ahora? } \\
\text { Describa esos factores que le dieron o le dan vida a la } \\
\text { Congregación para su mejor funcionamiento. }\end{array}$ \\
\hline 6. & $\begin{array}{l}\text { Imagine que usted está durmiendo, soñando y que al } \\
\text { despertar de ese sueño es el año 2018. Usted llega a la } \\
\text { Congregación Paulinas y encuentra que ha ocurrido un } \\
\text { milagro: todas y cada una de las mejores cualidades de } \\
\text { los colaboradores, de los cooperadores y de las hermanas } \\
\text { de la Congregación se han engrandecido, extendido y } \\
\text { multiplicado, y la Institución es exactamente todo aquello } \\
\text { que usted siempre quiso que fuera. ¿Cómo ha contribuido } \\
\text { usted en el logro de esa Congregación soñada? ¿Qué es } \\
\text { diferente, qué ha cambiado y cómo sabe que así es? }\end{array}$ \\
\hline 7. & $\begin{array}{l}\text { El mundo, la sociedad, nos está haciendo un constante } \\
\text { llamado a la transformación. ¿Cuáles son aquellas cosas que } \\
\text { independientemente de todas las transformaciones que } \\
\text { pueda sufrir la Congregación Paulinas le gustaría a usted } \\
\text { que perduraran en el futuro? }\end{array}$ \\
\hline
\end{tabular}

Fuente: elaboración propia.

Se realiza un prueba piloto de la encuesta aplicándola al $1 \%$ del personal de las librerías y como resultado se determina que se debe modificar: 1. agregar el encabezado y el objetivo a la encuesta; 2. adaptar la encuesta a cada uno de los grupos de interés con los que interactúa Paulinas; 3. clarificar el significado de lo social, ambiental y económico. 
La cantidad de encuestas que se aplican a los diferentes grupos de interés se representan en la Tabla 5 y las encuestas finalmente respondidas en la Tabla 6.

Tabla 5. Número de encuestas aplicadas

\begin{tabular}{|l|c|}
\hline \multicolumn{2}{|c|}{ ENCUESTAS APLICADAS } \\
\hline Colaboradores & 72 \\
\hline Hermanas & 30 \\
\hline Cooperadores & 30 \\
\hline Proveedores & 30 \\
\hline Destinatarios clientes & 150 \\
\hline Total & 312 \\
\hline
\end{tabular}

Fuente: elaboración propia.

Tabla 6. Número de encuestas respondidas

\begin{tabular}{|l|c|}
\hline \multicolumn{2}{|c|}{ ENCUESTAS RESPONDIDAS } \\
\hline Colaboradores & 41 \\
\hline Hermanas & 18 \\
\hline Cooperadores & 9 \\
\hline Proveedores & 4 \\
\hline Destinatarios clientes & 38 \\
\hline Total & 110 \\
\hline
\end{tabular}

Fuente: elaboración propia.

Algunas encuestas se aplican en encuentros en los que se explica previamente los conceptos de $\mathrm{RS}$ y luego se recogen los formatos ya diligenciados, como en el caso de los colaboradores y algunas hermanas. A otros grupos se les envía por correo electrónico, o se les entrega para que la envíen posteriormente, pero, esto mismo, dificulta y prolonga el proceso de recopilación de datos a pesar que se realizan seguimientos telefónicos o vía correo electrónico y visitas presenciales, por lo cual solo se recoge el $35 \%$ de las encuestas, cantidad que se considera como muestra representativa del total de la población de los grupos de interés de la Congregación y por consiguiente válida para obtener el diagnóstico y sacar conclusiones,

El análisis de los datos a la luz del EA permite evidenciar y comprobar en la Institución un comportamiento empresarialmente responsable pero aislado, que hace necesaria la construcción de un MRS, lo cual permite validar la hipótesis fenomenológica.

Con base en el consolidado obtenido de los grupos de interés, del respectivo análisis desde el enfoque apreciativo, y adicionalmente, de plantear los objetivos de la investigación como RAE según la taxonomía de Bloom, se propone un nuevo eje al modelo de sostenibilidad actual, el eje axiológico, visto no desde la enseñanza sino desde el aprendizaje, que responde a una de las principales necesidades detectadas y que posibilita un cambio de mentalidad para un trabajo mancomunado, propiciado desde los valores y desde talleres que faciliten el aprendizaje. Todo esto permite tener un panorama real y amplio con el cual se procede al diseño del MRS para Paulinas que se muestra en la Figura 1.

El modelo está soportado desde lo teórico por tres enfoques fundamentales: el Enfoque Apreciativo, el Enfoque Axiológico, y el Enfoque RAE; enlaza cíclicamente desde lo metodológico el Método Paulino (Camino, Verdad y Vida) y desde lo normativo los principios de RS de la Norma ISO 26000 y el ciclo PHVA. Además propone un cuarto eje al modelo actual de sostenibilidad, bajo el concepto de Desarrollo Sostenible, denominado "Cultura Organizacional inspirada en valores". Este eje es transversal y considera que el ser humano es un ente integral que se realiza en una existencia real, lo cual implica una posición axiológica siempre en favor de la vida; el hombre entendido de esta manera debe ser la referencia de todo valor. 
Figura 1. Modelo de Responsabilidad Social para Paulinas

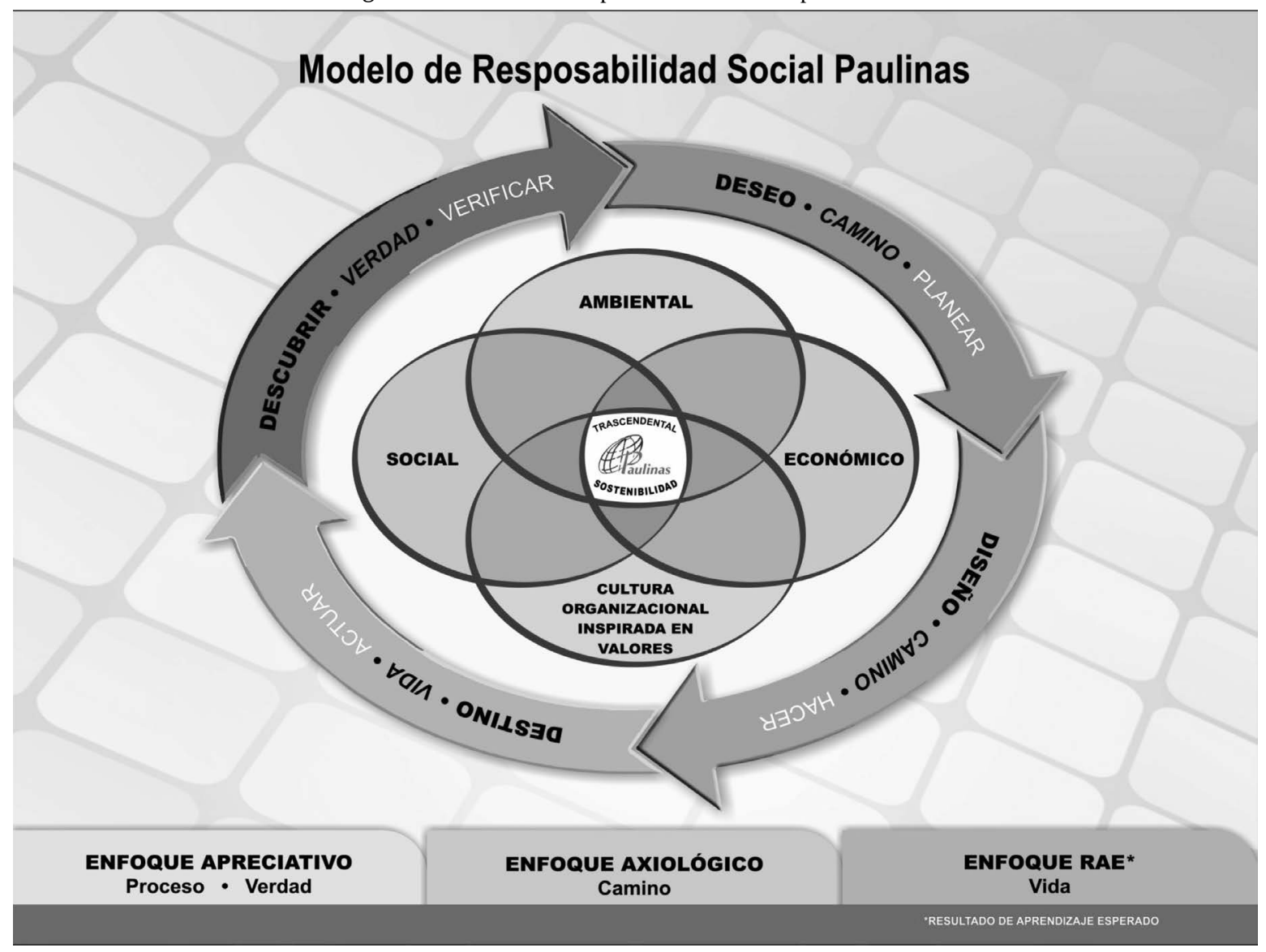

Fuente: elaboración propia, previa exploración de las fuentes.

Los valores constituyen el núcleo de toda cultura empresarial ya que aportan un sentido de direccionamiento común a todas las personas que componen la empresa y unas líneas orientadoras de su labor diaria. Los valores definen el carácter fundamental de la organización y crean sentido de identidad y de pertenencia a ella (Hamburger, 2004).

Según lo anterior, el cimiento de toda cultura organizacional son los valores. En tal sentido, estos conforman los principios éticos y el soporte filosófico del ser y quehacer de la empresa. Entendido así, los valores corporativos constituyen en gran medida el logro de los propósitos fundamentales de la empresa; es decir, de aquellos que giran en torno a la calidad, la productividad, el compromiso de los miembros de la empresa y la satisfacción de los clientes.

La Cultura organizacional es lo que identifica la manera de ser de la organización. Se manifiesta en la forma de actuación de sus miembros ante los problemas y oportunidades de gestión y adaptación a los cambios y requerimientos de orden exterior e interior. Debe ser interiorizada en forma de talantes y creencias colectivos que genera una manera de pensar, vivir y actuar, y que se transmite y se enseña a los nuevos integrantes de la organización.

Cabe resaltar que el ciclo PHVA no se aplica linealmente en la propuesta del modelo de Paulinas. La Tabla 7 muestra la relación que se establece entre las fases del EA, el método Paulino y el ciclo PHVA. 
Tabla 7. Relación entre el Enfoque Apreciativo,el método Paulino y el ciclo de la gestión PHVA

\begin{tabular}{|c|c|c|c|c|c|}
\hline \multicolumn{2}{|c|}{ CICLO ENFOQUE APRECIATIVO } & \multicolumn{2}{|c|}{$\begin{array}{l}\text { CICLO Ó MÉTODO PAULINO Cf. } \\
\text { Juan } 14,6\end{array}$} & \multicolumn{2}{|r|}{ CICLO PHVA } \\
\hline 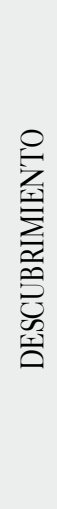 & $\begin{array}{l}\text { Se aprecia el sistema, se descubre } \\
\text { aquello que está funcionando, } \\
\text { resaltando lo mejor de las } \\
\text { experiencias. }\end{array}$ & 㓪 & $\begin{array}{l}\text { Término "emuná significa } \\
\text { primariamente "confianza", } \\
\text { "fidelidad" y del griego Alétheia, } \\
\alpha \lambda \eta \theta \varepsilon i \alpha \text {, concepto filosófico } \\
\text { que se refiere a la sinceridad } \\
\text { y veracidad de los hechos y la } \\
\text { realidad. Literalmente la palabra } \\
\text { significa "aquello que no está } \\
\text { oculto", "aquello que es evidente", } \\
\text { "lo que es verdadero". También } \\
\text { hace referencia al "desocultamiento } \\
\text { del ser". }\end{array}$ & $\begin{array}{l}\text { 壳 } \\
\text { 空 } \\
\text { 空 }\end{array}$ & $\begin{array}{l}\text { Realizar el seguimiento y la } \\
\text { medición de los procesos y } \\
\text { productos con base en los } \\
\text { objetivos y requisitos e informar } \\
\text { oportunamente los resultados. } \\
\text { ¿SE ESTÁN CUMPLIENDO } \\
\text { LOS PLANES? }\end{array}$ \\
\hline 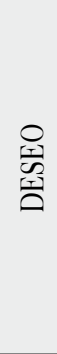 & $\begin{array}{l}\text { Se plantea el funcionamiento } \\
\text { ideal del sistema, lo que el mundo } \\
\text { demanda de él, articulado por } \\
\text { medio de tres cosas básicas: la } \\
\text { visión de un mundo mejor, un } \\
\text { propósito poderoso y la compilación } \\
\text { de estatutos de intentos } \\
\text { estratégicos. }\end{array}$ & 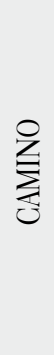 & 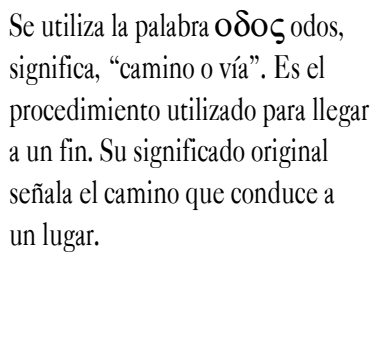 & $\begin{array}{l}\text { 总 } \\
\text { 㫘 } \\
\text { 至 }\end{array}$ & $\begin{array}{l}\text { Establecer los objetivos y procesos } \\
\text { necesarios para conseguir los } \\
\text { resultados esperados. } \\
\text { QUÉ PARA QUÉ } \\
\text { CUÁNDO } \\
\text { CÓMO } \\
\text { QUIÉN } \\
\text { CON QUÉ }\end{array}$ \\
\hline 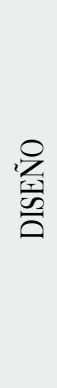 & $\begin{array}{l}\text { Construcción del sistema ideal, } \\
\text { la estructura social o el diseño } \\
\text { actual del sistema en relación con } \\
\text { el mundo del que hace parte; se } \\
\text { plantean las habilidades con las } \\
\text { cuales es posible alcanzar el sueño, } \\
\text { el camino se dibuja en equipo y } \\
\text { con experiencias del pasado que } \\
\text { resaltan recursos y fortalezas. }\end{array}$ & $\underset{⿱}{\stackrel{0}{ٍ}}$ & 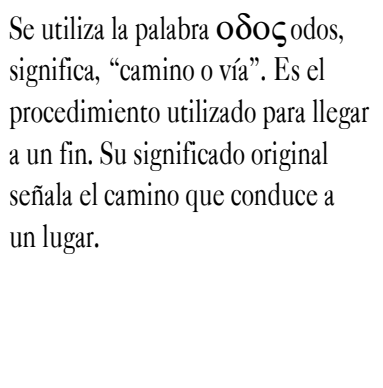 & $\begin{array}{l}\text { 琍 } \\
\text { 志 }\end{array}$ & $\begin{array}{l}\text { Implementar, realizar lo } \\
\text { planeado, hacer los procesos e } \\
\text { identificar las oportunidades } \\
\text { de mejora, implementarlas. } \\
\text { Ejecutar según lo planificado. } \\
\text { Generación de productos, servicio, } \\
\text { capacitación. }\end{array}$ \\
\hline 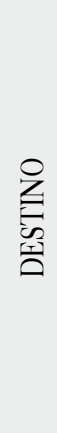 & $\begin{array}{l}\text { Se considera la forma de } \\
\text { empoderar, aprender y ajustar el } \\
\text { funcionamiento de la dinámica } \\
\text { que se está llevando a cabo; se } \\
\text { complejiza la mirada y se camina } \\
\text { desde el cambio hacia el cambio. }\end{array}$ & $\stackrel{\Xi}{ٍ}$ & 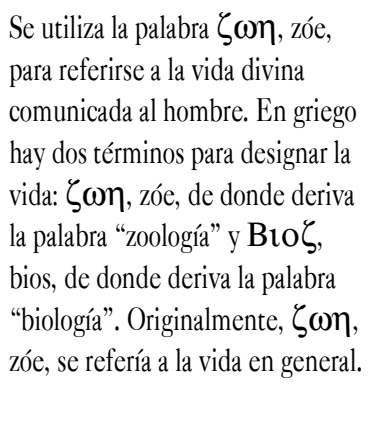 & 妥 & $\begin{array}{l}\text { Tomar acciones para } \\
\text { mejorar continuamente el } \\
\text { desempeño de los procesos. } \\
\text { Cómo mejorar. Acciones } \\
\text { preventivas/correctivas } \\
\text { NO = AJUSTAR } \\
\text { REPLANIFICAR } \\
\text { SI = MANTENERSE HASTA } \\
\text { CUMPLIR OBJETIVOS. } \\
\text { ASEGURAR - APRENDER }\end{array}$ \\
\hline
\end{tabular}

Fuente: elaboración propia. 
Dada la necesidad de poner en práctica el MRS, se elabora una herramienta pedagógica que permita la transformación del modelo a recurso. Se obtiene una cartilla de RS para Paulinas denominada "Ruta de la Verdad, el camino y la vida", que contempla entre otros temas: RS empresarial, MRS de Paulinas, comportamiento social, ambiental y económico de Paulinas, principios y valores: personales, institucionales y de la norma ISO 26000, temas fundamentales de la RS (gobernanza organizacional, derechos humanos, etc.). Estos temas son tratados a través de diferentes talleres propuestos que consideran diferentes estrategias pedagógicas tales como: trabajo individual y por equipos, expresión oral y escrita, actividades creativas (dibujos, recortes, etc.), cuadros explicativos y comparativos, y dramatizaciones entre otras.

A fin de establecer la percepción sobre los talleres propuestos en la cartilla se realiza una prueba piloto y se presenta el MRS para Paulinas. Para la prueba piloto se reúne un grupo de colaboradores representantes de diferentes áreas de Paulinas, con quienes se lleva a cabo la aplicación de 6 talleres de la cartilla sobre RS.

\section{CONCLUSIONES}

Si bien los principios que orientan a la Congregación Paulinas como institución religiosa están fundamentados en la fuente misma de la Palabra de Dios, al aplicar un modelo de responsabilidad social (MRS) se acepta que los principios planteados por la guía ISO 26000 orientan específicamente su contribución al desarrollo sostenible y al bienestar de la sociedad y fortalecen sus vínculos con las partes interesadas.

La identificación de paradigmas, tomados como creencias o mitos, a favor o de resistencia frente a la RS en Paulinas, obtenidos a través de entrevistas semiestructuradas -diálogos-a laicos y hermanas, resultan ser una base sólida para formular posteriormente las preguntas correspondientes de la Encuesta Apreciativa.

La encuesta apreciativa, diseñada teniendo en cuenta cada una de las fases del enfoque apreciativo (EA): descubrimiento, deseo, diseño y destino, permite no solo obtener datos sobre lo mejor del talento humano de Paulinas y de la institución como tal, sino además establecer lo que no se está haciendo bien, las razones de ello, y trazar un plan de acción, esto es, el cambio que se pretende lograr y el compromiso adquirido para tal fin.

El MRS planteado para la Congregación Paulinas está soportado por unas bases fundamentadas en el enfoque apreciativo, que busca lo mejor de las personas y de las organizaciones; en el enfoque de los resultados de aprendizaje esperados, que orienta desde el aprendizaje lo que se pretende lograr en los grupos de interés con respecto a la RS; y en el enfoque axiológico, que desde la teoría de los valores asume los valores organizacionales como los principios generales por los cuales se rigen las empresas y sus miembros. Pero adicionalmente, el modelo enlaza los tres ejes tradicionales de la sostenibilidad, que constituyen la finalidad de la RS: la sostenibilidad social, la sostenibilidad ambiental y la sostenibilidad económica y el eje adicional propuesto por este estudio, de cultura organizacional inspirada en valores. La relación cíclica de las fases del EA, el ciclo PHVA y el método Paulino integrando los principios de la norma ISO 26000, dan al MRS una visión de unidad, identidad y proyección.

La cultura organizacional inspirada en valores es la "imagen corporativa" de la organización, que hace que todos sus integrantes piensen la organización de la misma manera, tengan los mismos valores, las mismas creencias y los mismos símbolos frente a la RS, con lo cual se pueden coordinar las acciones y los proyectos que generan cohesión interna e irradian una imagen 
sólida hacia el exterior. Es el "sello" común que la hace diferente del resto de las empresas y trabajadores del sector.

Lo descrito en los puntos anteriores hace del MRS una propuesta que, desde los enfoques planteados, ayuda a generar procesos de concientización en todos los grupos de interés con los que la congregación interactúa y quienes posibilitan un trabajo conjunto para generar mayor impacto en la sociedad, aportar en la construcción de un mundo más humano, justo y solidario desde valores que reafirman su misión evangelizadora en la realidad de hoy.

La herramienta pedagógica que favorece la implementación del MRS en Paulinas es la cartilla de RS denominada "Ruta de la Verdad, el Camino y la Vida". La prueba piloto de los talleres allí planteados que enlazan e integran la visión personal, con lo institucional y normativo permite constatar un gran interés de los participantes en el tema de RS, por la metodología y dinámicas utilizadas en los mismos. El MRS causa impacto en los participantes y les es de fácil comprensión la presentación que del mismo se hace.

Se espera que la futura implementación del MRS le permita a la Congregación Paulinas incorporarlo en sus estrategias institucionales y desarrollar una estructura adecuada al ideal de evangelización que la institución está llamada a realizar en la sociedad, a través de metas y objetivos a mediano y largo plazo, cualificando cada día su misión evangelizadora. Asimismo, garantizar para Paulinas el crecimiento y la mejora de su gestión organizacional y la toma de conciencia de las implicaciones de la RS en cuanto a lo ambiental, social y económico como un proceso sostenible y de mejora continua para la institución y todos los grupos de interés con los que interactúa.

\section{REFERENCIAS}

Benedicto XVI. (2009). Carta encíclica, Caritas in Veritate, Bogotá: Paulinas, $1^{\text {a }}$ Ed.

Berger, P. y Luckmann, T. (2001). La construcción social de la realidad (p. 238). Buenos Aires: Amorrortu Editores S.A.

Cooperrider, D. y Whitney, D. (2005). Enfoque apreciativo: una revolución positiva en cambio. Michigan. Berrett- Koehler Publishers.

Corredor, C. y Misas, G. (2001). Desarrollo económico y social en Colombia: siglo XX. ISBN 9587010248. Bogotá: Universidad Nacional de Colombia.

Cortina, A. (1998). Ética de la empresa: Claves para una nueva cultura empresarial (p. 150). Madrid: Editorial Trotta.

Declaración de Río sobre el Medio Ambiente y el Desarrollo. Recuperado de http://www.un.org/esa/ dsd/agenda21_spanish/res_riodecl.shtml

Hamburger, Á. (2004). Ética de la Empresa, el Desafío de una Nueva Cultura Empresarial (p. 57). Bogotá: Paulinas.

Hammersley, M., Atkinson, P. (1994). Etnografía. Métodos de Investigación. Buenos Aires: Ediciones Paidós Ibérica S.A.

Hammond, S. (1996). The Thin Book of Appreciative Inquiry (p. 56). Bend: Thin Book Publishing Co.

Instituto Uruguayo de Normas Técnicas. (2010). Guía de Responsabilidad Social - UNIT- ISO 26000: 2010 IDT. Montevideo.

Juan Pablo II. (2004) Carta encíclica, Centesimus Annus, $7^{a}$ Ed. Bogotá: Paulinas. 
Juan XXIII. (1986). Carta encíclica, Pacem in Terris, $12^{\mathrm{a}}$ Ed. Bogotá: Paulinas.

Juan XXIII. (2004). Carta Encíclica, Mater et Magistra, $17^{\mathrm{a}}$ Ed. Bogotá: Paulinas.

Kennedy, D. (2007). Redactar y utilizar resultados de aprendizaje, Irlanda: University College Cork.

Krick, T., Forstater, M., Monaghan, P. y Sillanpää, M. (2006). De las palabras a la acción, el compromiso con los stakeholders. Manual para la práctica de las relaciones con los grupos de interés. Canadá: AccountAbility, United Nations Environment Programme.

Le Moigne, J. (1995). Les Épistemologies Constructivistes, Que sais-je? París: Presses Universitaires de France.
Pablo VI. (1994). Carta encíclica, Populorum Progressio, Bogotá: $18^{a}$ Ed. Paulinas.

Pablo VI. (2003). Carta encíclica, Gaudium et Spes, Bogotá: $16^{\mathrm{a}}$ Ed. Paulinas.

Pacto Mundial de las Naciones Unidas. Recuperado de https://www.unido.org/fileadmin/media/ documents/pdf/Procurement/Global_Compact/ GC_Brochure_Spanish.PDF

Pío XI. (2008). Carta Encíclica, Quadragesimo Anno, $1^{a}$ Ed. Bogotá: Paulinas.

Varona, F. (2007). La intervención apreciativa: una nueva manera de descubrir, crear, compartir e implementar conocimiento para el cambio en instituciones gubernamentales o privadas. Investigación y desarrollo vol. 15, n. 2 . 